\begin{tabular}{|c|c|c|}
\hline Beitr. Ent. & Keltern & ISSN 0005-805X \\
\hline $\mathbf{6 2}(2012) 1$ & S. $247-258$ & 15.05 .2012 \\
\hline
\end{tabular}

\title{
Dr. Günther Petersen (25.08.1924 - 24.03.2012) zum Gedenken
}

Betroffen habe ich die Nachricht zur Kenntnis genommen, dass Günther Petersen am 24. März diesen Jahres verstorben ist.

Es soll an dieser Stelle darauf verzichtet werden, Biografisches auszuführen, da das schon aus Anlass des 65. und 70. Geburtstages in dieser Zeitschrift erfolgt ist (39 (1): 3-8; 1989; 44 (2): 259-260, 1994).

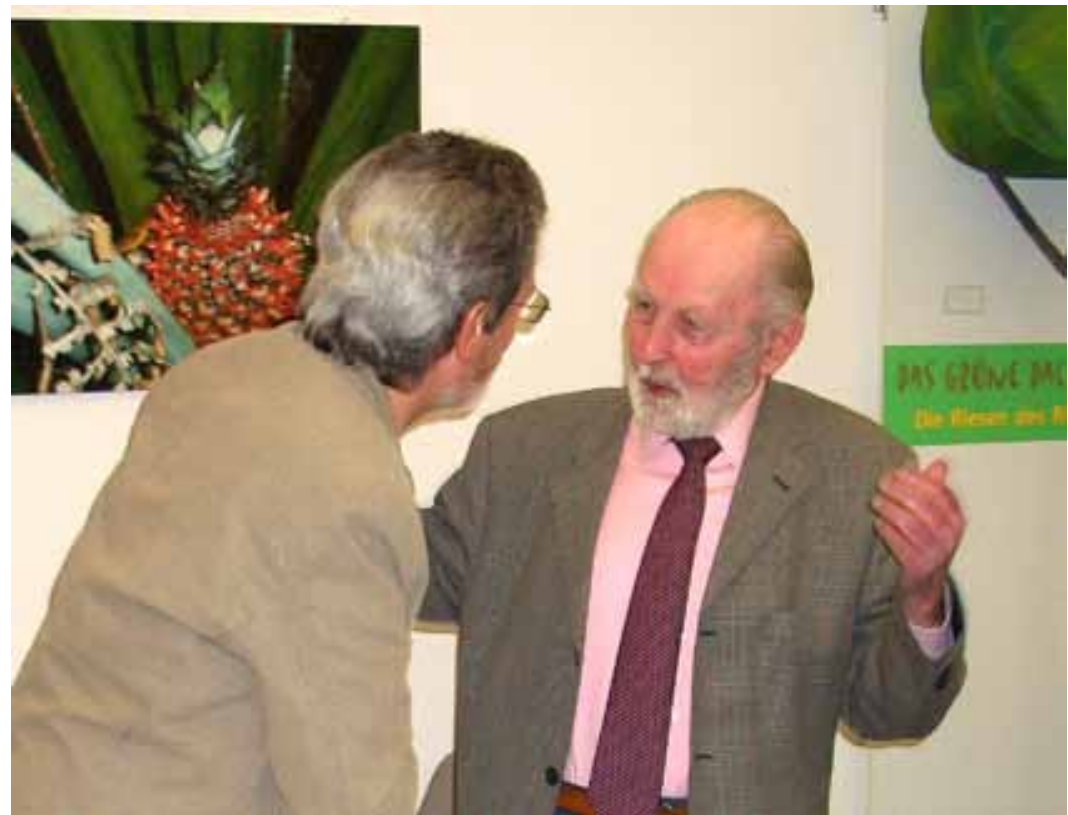

Dr. G. Petersen mit Dr. F. Menzel während der Feier zum 125jährigen Jubiläum des Deutschen Entomologischen Instituts am 13.12.2011 in Müncheberg, Foto: C. Kutzscher.

Mit seinem Tod verliert die Mikrolepidopterologie einen ihrer profilierten Vertreter. Über viele Jahrzehnte war er ein international anerkannter Spezialist für die Familie Tineidae. Seit der 1957 bis 1958 erschienenen Revision der europäischen Vertreter dieser Familie bestimmte sein Name mit die wissenschaftliche Entwicklung in dieser Gruppe. Ungezählte Falter aus Expeditionsausbeuten zahlreicher Museen und Sammler gingen durch seine Hände. Die Determinationszettel "Dr. G. Petersen det." zeugen heute beim Blick in viele Museumssammlungen von dieser Arbeit. Ein Ergebnis dieser Determinationen sind zahlreiche Neubeschreibungen, die einen wichtigen Erkenntniszuwachs bedeutet haben. Sie waren oft Teil umfangreicher Gattungs- und Gattungsgruppenrevisionen, wichtige Bausteine für die Familiensystematik. Bestechend an seinen Arbeiten waren neben den präzisen Formulierungen seine instruktiven Zeichnungen, die in ihrer Qualität unübertroffen sind. Ein weiteres wichtiges Ergebnis dieser jahrzehntelangen Arbeit ist die umfangreiche Datensammlung im DEI über die Systematik und Faunistik der Familie. Sie war die Grundlage für die Fortsetzung der wissenschaftlichen Bearbeitung der Tineidae durch mich. 
Seine Arbeit innerhalb der taxonomischen Abteilung des DEI bis zu seiner Pensionierung im Jahre 1989 hat das wissenschaftliche Profil des Instituts wesentlich geprägt. Auch wenn Günther Petersen aus gesundheitlichen Gründen danach nicht mehr wissenschaftlich tätig war, ist der Kontakt zum Institut nie abgerissen. Er war gern gesehener Gast bei zahlreichen Veranstaltungen des Hauses. Das letzte Mal konnten die Institutsmitarbeiter ihn Ende 2011 anlässlich der 125-Jahrfeier des DEI begrüßen.

Alle, die ihn kannten, werden ihn in bleibender Erinnerung behalten.

\section{Verzeichnis der Veröffentlichungen}

1. Petersen, G. 1953: Taxonomie und Verbreitung der “Kornmotten”. (Lepidoptera: Tineidae). - Beiträge zur Entomologie 3 (6): 577-600, 3 Fig.

2. Petersen, G. 1957: Zur systematischen Stellung zweier von L. Osthelder beschriebenen Microlepidopteren. (Lepidoptera: Acrolepiidae, Psychidae). - Nachrichtenblatt der Bayerischen Entomologen 6 (6): 62-63.

3. Petersen, G. 1957a: Die Genitalien der paläarktischen Tineiden [I] (Lepidoptera: Tineidae). - Beiträge zur Entomologie 7 (1/2): 55-176, Fig. 1-149, Taf. 1-4.

4. Petersen, G. 1957b: Die Genitalien der paläarktischen Tineiden [II] (Lepidoptera: Tineidae). - Beiträge zur Entomologie 7 (3/4): 338-379, Fig. 150-203, Taf. 6.

5. Petersen, G. 1957c: Die Genitalien der paläarktischen Tineiden [III] (Lepidoptera: Tineidae). - Beiträge zur Entomologie 7 (5/6): 557-595, Fig. 204-247, Taf. 11-12.

6. Petersen, G. 1957d: Kritische Bemerkungen über die Gattung Cilicorneola Zag. 1956 (Lepidoptera: Tineidae). - Beiträge zur Entomologie 7 (5/6): 596-598.

7. Petersen, G. 1958: Die Genitalien der paläarktischen Tineiden [IV] (Lepidoptera: Tineidae). - Beiträge zur Entomologie 8 (1/2): 111-118, Fig. 248-252.

8. Petersen, G. 1958a: Die Genitalien der paläarktischen Tineiden [V] (Lepidoptera: Tineidae). - Beiträge zur Entomologie 8 (3/4): 398-430, Fig. 253-267, Taf. 2-3.

9. Petersen, G. 1958b: Neue paläarktische Tineiden aus der Gruppe der lichenophagen Gattungen (Lepidoptera, Tineidae). - Deutsche entomologische Zeitschrift, N. F. 5 (3/4): 367-375, 12 Fig.

10. Petersen, G. 1959: Revision der Gattung Obesoceras Pet. 1957 (Lepidoptera, Tineidae) und Beschreibung einer neuen Art aus der Sammlung des Nationalmuseums in Prag. - Casopis Ceskoslovenské Spolecnosti Entomologiské 56 (2): 197-199, 2 Fig.

11. Petersen, G. 1959a: Ergebnisse der Untersuchung indeterminierter paläarktischer Tineiden aus dem Zoologischen Museum Berlin und der Sammlung H. G. Amsel / Karlsruhe (Lepidoptera). - Deutsche entomologische Zeitschrift, N. F. 6 (1/3): 152-159, 3 Fig.

12. Petersen, G. 1959b: Tineidae. - In: Amsel, H. G.: Irakische Kleinschmetterlinge II. - Bulletin de la Société Entomologique d'Égypte 43: 70-72.

13. Petersen, G. 1959c: Tineiden aus Afghanistan mit einer Revision der paläarktischen Scardiinen (Lepidoptera: Tineidae). - Beiträge zur Entomologie 9 (5/6): 558-579, 27 Fig., Taf. 32.

14. Petersen, G. 1959d: Un nuevo Nemapogon Schrk., Espanol (Lepidoptera, Tineidae). - Archivos del Instituto de Aclimatacion 8: 45-46, 2 Fig.

15. Petersen, G. 1960: Zwei neue paläarktische Tineiden aus dem Iran (Lepidoptera). - Stuttgarter Beiträge zur Naturkunde Nr. 34: 1-3, 2 Fig.

16. Petersen, G. 1960a: Die Monopis-Arten der rusticella-Gruppe (Lepidoptera: Tineidae). - Beiträge zur Entomologie 10 (3/4): 409-418, 4 Fig., Taf. 8-9.

17. Petersen, G. 1960b: Contribucion al conocimiento de la distribucion geografica de los Tineidos de la Peninsula Iberica (Lepidoptera, Tineidae). - Eos: Revista española de entomología 36 (2): 205-236, 8 Fig. 
18. Petersen, G. 1961: Tineiden aus SW-Arabien der Ausbeuten H. Scott und E. B. Britton 1937/38. Beiträge zur naturkundlichen Forschung in Südwestdeutschland 20 (1): 63-65, 3 Fig.

19. Petersen, G. 1961a: Zur Taxonomie und Verbreitung der paläarktischen Nemapogon-Arten (Lepidoptera, Tineidae). - Casopis Ceskoslovenské Spolecnosti Entomologické 58 (3): 272-283, 13 Fig.

20. Petersen, G. 1961b: Identity, Synonymy and generic position of Tinea confusella H.-S. (Lepidoptera, Tineidae). - Entomologist's Gazette 12 (3): 117-120, 5 Fig.

21. Petersen, G. 1961c: Zur Identität und generischen Stellung von "Tinea mendicella Нв.” und "Tinea piercella Bent.” (Lepidoptera: Tineidae). - Notulae Entomologicae 41: 80-85, 7 Fig.

22. Petersen, G. 1961d: Beitrag zur Kleinschmetterlingsfauna der Dresdener Umgebung (Tineidae, Lepidoptera). - Entomologische Abhandlungen, Abhandlungen und Berichte aus dem Staatlichen Museum für Tierkunde in Dresden 26 (7): 53-62.

23. Petersen, G. 1961e: Tineiden aus Riad (Zentral-Arabien) der Ausbeute von Dr. E. Diehl (Lepidoptera: Tineidae). - Beiträge zur naturkundlichen Forschung in Südwestdeutschland 20 (2): 107-110, 3 Fig.

24. Petersen, G. 1962: New and rare Tineids (Lepidoptera: Tineidae) in the Collections of the British Museum (Natural History). - Annals and Magazine of Natural History, Ser. 13, 4: 529-539, 18 Fig.

25. Petersen, G. 1962a: Hämozytäre Abwehrreaktionen des Wirtes gegen endoparasitische Insekten und ihre Bedeutung für die biologische Bekämpfung. - Bericht über die 9. Wanderversammlung Deutscher Entomologen 9: 179-194, 2 Fig.

26. Petersen, G. 1962b: Beitrag zur Kenntnis der südeuropäischen Tineiden (Lepidoptera, Tineidae). Beiträge zur naturkundlichen Forschung in Südwestdeutschland 21 (2): 205-220, 15 Fig.

27. Petersen, G. 1963: Ergebnisse der Albanien-Expedition 1961 des Deutschen Entomologischen Institutes. 3. Beitrag. Lepidoptera: Tineidae. - Beiträge zur Entomologie 13 (1/2): 1-20, 4 Fig.

28. Petersen, G. 1963a: Revision der paläarktischen Arten der Gattung Catabola Durr. (Lepidoptera: Tineidae). - Beiträge zur Entomologie 13 (1/2): 168-175, 12 Fig.

29. Petersen, G. 1963b: 2. Beitrag zur Kenntnis der Tineiden von Afghanistan (Lepidoptera: Tineidae). Beiträge zur Entomologie 13 (1/2): 176-188, 7 Fig.

30. Petersen, G. 1963c: Tineiden als Bestandteil der Nidicolenfauna (Lepidoptera: Tineidae). - Beiträge zur Entomologie 13 (3/4): 411-427.

31. Petersen, G. 1964: Über einige Tineiden aus SW-Europa. - Reichenbachia, Staatliches Museum für Tierkunde in Dresden 2 (58): 225-233, 10 Fig.

32. Petersen, G. 1964a: Neue und seltene lichenophage Tineiden aus Südeuropa (Lepidoptera: Tineidae). - Nachrichtenblatt der Bayerischen Entomologen 13 (3): 17-25, 5 Fig.

33. Petersen, G. 1964b: Beitrag zur Schmetterlingsfauna Thüringens, des Harzes und der Umgebung von Halle/Saale (Lepidoptera: Tineidae). - Faunistische Abhandlungen Staatliches Museum für Tierkunde in Dresden 1 (3): 111-126.

34. Petersen, G. 1964c: Ergebnisse der Albanien-Expedition 1961 des Deutschen Entomologischen Institutes. 29. Beitrag. Lepidoptera: Psychidae I (Micropsychina sensu Dalla Torre \& Strand, 1929). - Beiträge zur Entomologie 14 (3/4): 377-380.

35. Petersen, G. 1964d: Zur systematischen Stellung von “Tinea moeniella Rössler, 1877” (Lepidoptera: Tineidae). - Beiträge zur Entomologie 14 (3/4): 391-393.

36. Petersen, G. 1964e: Zweiter Beitrag zur Kenntnis der geographischen Verbreitung der Tineiden auf der Iberischen Halbinsel (Lepidoptera: Tineidae). - Beiträge zur Entomologie 14 (3/4): 395-420, 10 Fig.

37. Petersen, G. 1964f: Zur Erforschung der Kleinschmetterlingsfauna in Nestern von Vögeln, Säugern und sozialen Hymenopteren. - Entomologische Berichte 1963 (2)(1964): 77-80, 1 Fig.

38. Petersen, G. 1964g: Tineiden aus Karatschi (Pakistan), Fars (SW-Iran) und den Bahrein-Inseln (Lepidoptera: Tineidae). - Beiträge zur naturkundlichen Forschung in Südwestdeutschland 23 (2): 111-122, 11 Fig.

39. Petersen, G. 1964h: Zur Synonymie von Perissomastix Warren \& Rothschild, 1905 (Lepidoptera: Tineidae). - Beiträge zur Entomologie 14 (5/6): 625-629, 2 Fig. 
40. Petersen, G. 1965: Revision der Gattungen Rhodobates Ragonot und Pachyarthra Amsel (Lepidoptera: Tineidae). - Beiträge zur Entomologie 15 (1/2): 87-100, 23 Fig.

41. Petersen, G. 1965a: Der gegenwärtige Stand der Erforschung der Kleinschmetterlingsfauna der DDR. - Entomologische Nachrichten 9 (4): 45-55.

42. Petersen, G. 1965b: 56. Tineidae. Ergebnisse der zoologischen Forschungen von Dr. Z. Kaszab in der Mongolei (Lepidoptera) (Mit einer Revision der palaearktischen Myrmecozela-Arten). - Reichenbachia, Staatliches Museum für Tierkunde in Dresden 7 (12): 107-111, 8 Fig.

43. Petersen, G. 1965c: Beitrag zur Kleinschmetterlingsfauna der Berliner Umgebung und der nördlichen Bezirke der DDR (Lepidoptera: Tineidae). - Faunistische Abhandlungen Staatliches Museum für Tierkunde in Dresden 1 (5): 221-230.

44. Petersen, G. 1965d: Beitrag zur Kenntnis der Tineiden der Tschechoslowakei (Lepidoptera: Tineidae). - Acta faunistica entomologica Musei Nationalis Pragae 11 (103): 165-194, 2 Fig.

45. Petersen, G. 1966: Über einige Tineiden aus Thüringen, gesammelt von Dr. H. Steuer. Entomologische Nachrichten 9 (2)(1965): 33-36, 2 Fig.

46. Petersen, G. 1966a: Die Microlepidopteren der Brandt'schen Iran-Ausbeute. 6. Teil: Tineidae. Entomologisk Tidskrift 87 (1/2): 23-29, 4 Fig.

47. Petersen, G. 1967: Zur Erforschung der Microlepidopterenfauna des mitteleuropäischen Raumes. - II. Entomologisches Symposium über die Probleme der faunistischen und entomogeographischen Erforschung der Tschechoslowakei und Mitteleuropas, September 1966. - Opava: 215-225.

48. Petersen, G. 1967a: Dritter Beitrag zur Kenntnis der geographischen Verbreitung der Tineiden auf der Iberischen Halbinsel (Lepidoptera: Tineidae). - Beiträge zur Entomologie 17 (3/4): 357-361, 3 Fig.

49. Petersen, G. 1968: Beitrag zur Kenntnis der ostmediterranen Tineiden (Lepidoptera: Tineidae, exclus. Nemapogoninae). - Acta entomologica bohemoslovaca 65 (1): 52-66, 15 Fig.

50. Petersen, G. 1968a: Verzeichnis der wichtigsten Sammlungen, die Microlepidopteren aus dem Gebiet der DDR enthalten (Arbeitsmaterialien für Kleinschmetterlingssammler, Nr. 2). - Entomologische Berichte 1968: 69-77.

51. Petersen, G. 1968b: Beitrag zur Kenntnis der Tineiden Westdeutschlands (Lepidoptera: Tineidae). Acta faunistica entomologica Musei Nationalis Pragae 13 (145): 87-107.

52. Petersen, G. \& Gaedike, H. 1968c: Katalog der in den Sammlungen des Deutschen Entomologischen Institutes aufbewahrten Typen - I (Ephemeroptera, Odonata, Plecoptera). - Beiträge zur Entomologie 18 (7/8): 959-969.

53. Petersen, G. 1969: Primäres Nahrungssubstrat und sekundäres Schadauftreten bei Mottenlarven (Lepidoptera: Tineidae). - Berichte der Wanderversammlung Deutscher Entomologen 10: 223-229.

54. Petersen, G. 1969a: Beiträge zur Insektenfauna der DDR: Lepidoptera - Tineidae. - Beiträge zur Entomologie 19 (3/6): 311-388, 205 Fig., 44 Farbabb.

55. Petersen, G. \& Gaedike, H. 1970: Katalog der in den Sammlungen des Deutschen Entomologischen Institutes aufbewahrten Typen - II (Dermaptera, Mantodea, Blattariae, Isoptera, Phasmida, Saltatoria). - Beiträge zur Entomologie 20 (1/2): 145-172.

56. Petersen, G. 1971: Beitrag zur Kenntnis der Tineiden von Iran und Pakistan (Lepidoptera: Tineidae). - Beiträge zur Entomologie 21 (3/6): 267-271, 2 Fig.

57. Petersen, G. 1971a: Zur entomologischen Forschung im Grenzgebiet zwischen der DDR und der VR Polen in den letzten 25 Jahren. - Polskie Pismo Entomologiczne 41 (4): 887-893.

58. Petersen, G. 1973: 234. Tineidae II. Ergebnisse der zoologischen Forschungen von Dr. Z. Kaszab in der Mongolei (Lepidoptera). - Reichenbachia, Staatliches Museum für Tierkunde in Dresden 14 (11): 89-94.

59. Petersen, G.; Friese, G. \& Rinnhofer, G. 1973a: Beiträge zur Insektenfauna der DDR: Lepidoptera Crambidae. - Beiträge zur Entomologie 23 (1-4): 4-55, 42 Fig., 51 Farbabb.

60. Petersen, G. 1973b: Dritter Beitrag zur Kenntnis der Tineiden von Afghanistan (Lepidoptera: Tineidae). - Beiträge zur Entomologie 23 (1/4): 57-69, 41 Fig. 
61. Petersen, G. 1973c: Beiträge zur Insektenfauna der DDR: Lepidoptera-Galleriidae. - Beiträge zur Entomologie 23 (5/8): 313-324, 25 Fig., 2 Taf.

62. Petersen, G. \& Gaedike, R. 1975: Beiträge zur Insektenfauna der DDR: Lepidoptera, Nachträge I (Tineidae, Epermeniidae, Acrolepiidae). - Entomologische Berichte 1975: 75-79, 1 Fig.

63. Petersen, G. 1978: Zur systematischen Stellung der Gattung Crinopteryx Peyerimhoff, 1871 (Lepidoptera: Incurvariidae). - Beiträge zur Entomologie 28 (2): 217-220, 12 Fig.

64. Petersen, G. 1979: Eine neue Monopis-Art aus Österreich (Lepidoptera, Tineidae). - Entomologische Nachrichten 23 (3): 35-38, 6 Fig.

65. Petersen, G. 1979a: Revision der von Conte Emilio Turati beschriebenen Tineiden (Lepidoptera: Tineidae). - Beiträge zur Entomologie 29 (2): 373-381, 2 Fig.

66. Petersen, G. \& Gaedike, R. 1979b: Beitrag zur Kenntnis der Tineiden-Fauna des Mittelmeerraumes. - Beiträge zur Entomologie 29 (2): 383-412, 29 Fig.

67. Petersen, G. 1980: Tineid moths from Tunisia (Lepidoptera: Tineidae). - Folia entomologica Hungarica 41 (1): $145-148$.

68. Petersen, G. \& Gaedike, R. 1980a: Zur Taxonomie der einheimischen Dioryctria-Arten (Lepidoptera, Phycitidae). - Entomologische Berichte 1980 (1): 21-35, 31 Fig.

69. Petersen, G. 1982: Tineiden aus Nepal (Lepidoptera, Tineidae). - Reichenbachia, Staatliches Museum für Tierkunde in Dresden 20 (8): 73-76, 10 Fig.

70. Petersen, G. \& Gaedike, R. 1982a: Insects of Saudi Arabia. Lepidoptera: Tineidae. - Fauna of Saudi Arabia 4: 333-346, 47 Fig.

71. Petersen, G. 1983: Revision der Gattung Triaxomera Zagulajev mit Bemerkungen zur Phylogenie der Nemapogoninae (Lepidoptera, Tineidae). - Entomologische Abhandlungen Staatliches Museum für Tierkunde in Dresden 46 (9): 177-196, 27 Fig.

72. Petersen, G. 1983a: Revision der Gattung Dinica Gozmány (Lepidoptera, Tineidae). - Entomologische Abhandlungen Staatliches Museum für Tierkunde Dresden 47 (3): 35-41, 16 Fig.

73. Petersen, G. \& Gaedike, R. 1983b: Beiträge zur Insektenfauna der DDR: Lepidoptera, Nachträge III (Epermeniidae, Tineidae, Acrolepiidae, Crambidae, Pyralidae, Pyraustinae, Scopariinae). Entomologische Nachrichten und Berichte 27 (1): 1-8, 12 Fig.

74. Petersen, G. \& Gaedike, R. 1983c: Records of the Lepidoptera of Greece based on the collections of G. Christensen and L. Gozmany: VII, Tineidae, Epermeniidae, Acrolepiidae, Douglasiidae. Annales Musei Goulandris 6: 271-311, 17 Fig.

75. Petersen, G. 1984: Grundlagen der Inventarerkundung der Oberlausitzer Kleinschmetterlinge. Abhandlungen und Berichte des Naturkundemuseums Görlitz 58 (2): 49-60, 2 Fig.

76. Petersen, G. \& Gaedike, R. 1984a: Beitrag zur Kenntnis der Tineidenfauna des Vorderen und Mittleren Orients (Tineidae, Lep.). - Acta faunistica entomologica Musei Nationalis Pragae 17 (202): 185-212, 30 Fig.

77. Petersen, G. 1984b: Eine neue Triaxomera-Art aus dem Parco Nazionale d'Abruzzo (Italien) (Lepidoptera, Tineidae). - Reichenbachia, Staatliches Museum für Tierkunde Dresden 22 (18): 141-145, 6 Fig.

78. Petersen, G. \& Gaedike, R. 1985: Die Microlepidopteren Zyperns. 2. Teil: Tineidae (Lepidoptera). Reichenbachia, Staatliches Museum für Tierkunde Dresden 23 (7): 29-34, 12 Fig.

79. Petersen, G. \& Gaedike, R. 1985a: Beitrag zur Kleinschmetterlingsfauna Österreichs und der angrenzenden Gebiete (Lepidoptera: Tineidae, Epermeniidae, Acrolepiidae, Douglasiidae). - Mitteilungen der Abteilung für Zoologie am Landesmuseum Joanneum 36: 1-48.

80. Gaedike, R. \& Petersen, G. 1985b: Beiträge zur Insektenfauna der DDR: Lepidoptera - Phycitidae. - Faunistische Abhandlungen Staatliches Museum für Tierkunde Dresden 13 (4): 55-107, 128 Fig.

81. Ebert, W.; Rohlfien, K.; Petersen, G. \& Friese, G. 1986: Einhundert Jahre Deutsches Entomologisches Institut. - Beiträge zur Entomologie 36 (1): 5-52, 25 Fig. 
82. Petersen, G. 1986a: Revision der Gattung Gephyristis Meyrick (Lepidoptera: Tineidae, Hieroxestinae). - Beiträge zur Entomologie 36 (1): 53-62, 24 Fig.

83. Petersen, G. 1986b: Nomenklatorische Bemerkungen zu den von Herrich-Schäffer beschriebenen Tineiden (Lepidoptera). - Beiträge zur Entomologie 36 (1): 69-74, 1 Fig.

84. Petersen, G. \& Gaedike, R. 1987: Beiträge zur Insektenfauna der DDR: Lepidoptera Nachträge IV (Tineidae, Acrolepiidae, Crambidae, Galleriidae, Pyralidae s. str., Douglasiidae, Pyraustinae, Scopariinae, Phycitinae). - Entomologische Nachrichten und Berichte 31 (1): 29-36.

85. Petersen, G. 1987a: A new Gerontha (Tineidae) from China. - Tinea, Supplement 12: 152-154, 7 Fig.

86. Petersen, G. 1987b: Revision der Gattung Rhodobates Ragonot (Lepidoptera, Tineidae, Hapsiferinae). - Entomologische Abhandlungen Staatliches Museum für Tierkunde Dresden 50 (9): 167-190, 67 Fig.

87. Petersen, G. 1988: Revision der Perissomasticini (Lepidoptera: Tineidae). - Beiträge zur Entomologie 38 (1): 3-64, 234 Fig.

88. Petersen, G. 1988a: Revision der Gattung Ateliotum Zeller, 1839 (Lepidoptera: Tineidae). - Beiträge zur Entomologie 38 (2): 311-324, 53 Fig.

89. Petersen, G. \& Gaedike, R. 1990: Nachtrag zur Lepidopterenfauna Griechenlands (Tineidae, Epermeniidae, Acrolepiidae, Douglasiidae). - Annales Musei Goulandris 8: 291-307.

90. Petersen, G. 1991: Zur Taxonomie und Verbreitung der Hapsiferinae (Lepidoptera, Tineidae). Deutsche entomologische Zeitschrift, N. F. 38 (1/3): 27-33, 10 Fig.

91. Petersen, G. \& Gaedike, R. 1992: Artenbestand und geographische Verbreitung der Tineiden der Iberischen Halbinsel (Lepidoptera: Tineidae). - Shilap Revista de Lepidopterologia 20 (80): 325-353, 9 Fig.

92. Petersen, G. \& Gaedike, R. 1993: Tineiden aus China und Japan aus der Höne-Sammlung des Museums Koenig (Lepidoptera: Tineidae). - Bonner zoologische Beiträge 44 (3/4): 241-250, 14 Fig.

93. Petersen, G. \& Gaedike, R. 1996: Tineidae. - In: Karsholt, O. \& Razowski, J. (eds.): The Lepidoptera of Europe. - A Distributional Checklist. - Apollo Books Aps. Stenstrup: 31-38.

94. Petersen, G. \& Gaedike, R. 1996a: Revision der Gattung Neurothaumasia LeMarchand, 1934 (Insecta: Lepidoptera: Tineidae). - Entomologische Abhandlungen Staatliches Museum für Tierkunde Dresden 57 (13): 283-294, 32 Fig.

\section{Gattungs- und Artbeschreibungen von Günther Petersen}

\section{Gattungen}

Betroka Petersen, 1988 (nec Betroka Viette, 1955) Beiträge zur Entomologie 38 (1): 27; jetzt: Trokabe VIETTE, 1993

Ceratophaga Petersen, 1957 Beiträge zur Entomologie 7 (1/2): 130 - 131

Ceratuncus Petersen, 1957 Beiträge zur Entomologie 7 (1/2): 105

Denticlunicula Petersen, 1988 Beiträge zur Entomologie 38 (1): 12

Lichenotinea Petersen, 1957 Beiträge zur Entomologie 7 (3/4): 371 - 372

Lichenovora Petersen, 1957 Beiträge zur Entomologie 7 (3/4): 344; jetzt: Tenaga Clemens, 1862

Metatinea Petersen \& Gaedike, 1979 Beiträge zur Entomologie 29 (2): 407-408

Montetinea Petersen, 1957 Beiträge zur Entomologie 7 (3/4): 342 - 343

Morophagoides Petersen, 1957 Beiträge zur Entomologie 7 (5/6): 593

Neoepiscardia Petersen \& GAedike, 1982 Fauna of Saudi Arabia 4: 336; jetzt: Edosa WALker, 1866 
Neomeessia Petersen, 1968 Acta entomologica bohemoslovaca 65 (1): 58; jetzt: Eudarcia Clemens, 1860

Neopsolarcha Petersen, 1988 Beiträge zur Entomologie 38 (1): 26

Niditinea Petersen, 1957 Beiträge zur Entomologie 7 (1/2): 134

Obesoceras Petersen, 1957 Beiträge zur Entomologie 7 (3/4): 352; jetzt: Eudarcia Clemens, 1860

Pararhodobates Petersen, 1958 Beiträge zur Entomologie 8 (3/4): 404

Paratinea Petersen, 1957 Beiträge zur Entomologie 7 (1/2): 159; jetzt: Proterospastis Meyrick, 1937

\section{Arten}

afghana Petersen, 1959 (Catabola) Beiträge zur Entomologie 9 (5/6): 562; jetzt: Praelongicera afghana (Petersen, 1959)

agenjoi Petersen, 1959 (Nemapogon) Archivos del Instituto de Aclimatacion 8: 45-46

albanica Petersen, 1963 (Infurcitinea) Beiträge zur Entomologie 13 (1/2): 17-18

albarracinella Petersen, 1967 (Novotinea) Beiträge zur Entomologie 17(3/4): 360

almaella Petersen, 1957 (Fermocelina) Beiträge zur Entomologie 7 (1/2): 118-119; jetzt: Anomalotinea almaella (Petersen, 1957)

alpicella Petersen, 1962 (Infurcitinea) The Annals and Magazine of natural History Series 13, 4 (9)(1961): 535-536; jetzt: Infurcitinea roesslerella (HEYDEN, 1865)

amseli Petersen, 1959 (Catabola) Beiträge zur Entomologie 9 (5/6): 561; jetzt: Perissomastix amseli (Petersen, 1959)

amseli Petersen \& Gaedike, 1982 (Episcardia) Fauna of Saudi Arabia 4: 336; jetzt: Edosa amseli (Petersen \& Gaedike, 1982)

amseli Petersen, 1957 (Infurcitinea) Beiträge zur Entomologie 7 (3/4): 357-358

anatolica Petersen, 1968 (Infurcitinea) Acta entomologica bohemoslovaca 65 (1): 64-65

andalusiella Petersen, 1964 (Novotinea) Beiträge zur Entomologie 14 (3/4): 408-409

arabica Petersen, 1961 (Fermocelina) Beiträge zur naturkundlichen Forschung in Südwestdeutschland 20 (2): 109-110; jetzt: Reisserita arabica (Petersen, 1961)

arabicum Petersen, 1961 (Ateliotum) Beiträge zur naturkundlichen Forschung in Südwestdeutschland 20 (1): 65

arenbergeri Petersen \& Gaedike, 1985 (Ateliotum) Reichenbachia Staatliches Museum für Tierkunde Dresden 23 (7): 33

asiatica Petersen, 1959 (Pachyarthra) Beiträge zur Entomologie 9 (5/6): 559

asiriella Petersen \& Gaedike, 1982 (Perissomastix) Fauna of Saudi Arabia 4: 338, 340

barikotellus Petersen, 1973 (Nemapogon) Beiträge zur Entomologie 23 (1/4): 57-58

baldensis Petersen, 1983 (Triaxomera) Entomologische Abhandlungen Staatliches Museum für Tierkunde in Dresden 46 (9): 177-180

bifurcatella Petersen, 1957 (Catabola) Beiträge zur Entomologie 7 (5/6): 569; jetzt: Crassicornella bifurcatella (Petersen, 1957)

brandti Petersen, 1966 (Pachyarthra) Entomologisk Tidskrift 87 (1/2): 24-25

brunneopterella PeTERSEN, 1964 (Infurcitinea) Beiträge zur naturkundlichen Forschung in Südwestdeutschland 23 (2): 118-119 
burmanni Petersen, 1979 (Monopis) Entomologische Nachrichten 1979 (3): 35-38

canariensis Petersen \& Gaedike, 1979 (Rhodobates) Beiträge zur Entomologie 29 (2): 397

christophi Petersen, 1957 (Fermocelina) Beiträge zur Entomologie 7 (1/2): 117-118; jetzt: Anomalotinea liguriella (MiluIÈre, 1879)

christophi Petersen, 1957 (Monopis) Beiträge zur Entomologie 7 (1/2): 170-171

confusum Petersen, 1966 (Ateliotum) Entomologisk Tidskrift 87 (1/2): 25-26

cornuta Petersen, 1959 (Catabola) Bulletin de la Société entomologique d'Égypte 43: 70-71; jetzt: Persissomastix cornuta (Petersen, 1959)

croaticum PeTERSEN, 1962 (Obesoceras) Beiträge zur naturkundlichen Forschung in Südwestdeutschland 21 (2): 210-212; jetzt: Eudarcia croatica (Petersen, 1962)

cyprica Petersen \& Gaedike, 1985 (Infurcitinea) Reichenbachia Staatliches Museum für Tierkunde Dresden 23 (7): 31-32

danubiellum Petersen, 1959 (Obesoceras) Časopis Československé Společnosti entomologické 56 (2): 197-198; jetzt: Eudarcia confusella (ZELLER, 1852)

dierli Petersen, 1983 (Dinica) Entomologische Abhandlungen Staatliches Museum für Tierkunde in Dresden 47 (3): 36-39

distinguenda Petersen, 1957 (Niditinea) Beiträge zur Entomologie 7 (1/2): 136; jetzt: Niditinea striolella (MATSUMURA, 1931)

echinatum Petersen \& Gaedike, 1985 (Obesoceras) Reichenbachia Staatliches Museum für Tierkunde Dresden 23 (7): 32; jetzt: Eudarcia echinata (Petersen \& Gaedike, 1985)

egregiellum Petersen, 1973 (Obesoceras) Beiträge zur Entomologie 23 (1/4): 58; jetzt: Eudarcia egregiella (Petersen, 1973)

falcata Petersen, 1988 (Perissomastix) Beiträge zur Entomologie 38 (1): 9

fasciata Petersen, 1959 (Neurothaumasia) Beiträge zur Entomologie 9 (5/6): 565-566

fasciella Petersen \& Gaedike, 1984 (Infurcitinea) Acta faunistica entomologica Musei Nationalis Pragae 17 (202): 191, jetzt: Infurcitinea fasciella GAEDIKE, 1983

flava Petersen, 1960 (Catabola) Stuttgarter Beiträge zur Naturkunde Nr. 34: 2-3; jetzt: Perissomastix (Aphrodoxa) flava (Petersen, 1960)

flavifrons Petersen, 1959 (Nemapogon) Beiträge zur Entomologie 9 (5/6): 565

forsteri Petersen, 1964 (Obesoceras) Nachrichtenblatt der Bayerischen Entomologen 13 (3): 17-18; jetzt: Eudarcia forsteri (PeTERSEN, 1964)

friedeli Petersen, 1987 (Rhodobates) Entomologische Abhandlungen Staatliches Museum für Tierkunde Dresden 50 (9): 172-173

gallica Petersen, 1962 (Meessia) The Annals and Magazine of natural History Series 13, 4 (9) (1961): 534-535; jetzt: Eudarcia gallica (Petersen, 1962)

glaseri Petersen, 1967 (Obesoceras) Beiträge zur Entomologie 17 (3/4): 358-360; jetzt: Eudarcia glaseri (Petersen, 1967)

gomerae, ssp. von pinkeri Petersen, 1987 (Rhodobates) Entomologische Abhandlungen Staatliches Museum für Tierkunde Dresden 50 (9): 174

gracilis Petersen, 1968 (Neomeessia) Acta entomologica bohemoslovaca 65 (1): 58; jetzt: Eudarcia gracilis (Petersen, 1968) 
graeca Petersen \& Gaedike, 1983 (Infurcitinea) Annales Musei Goulandris 6: 285-286, jetzt: Infurcitinea graeca GAEDIKE, 1983

gravosaellus Petersen, 1957 (Nemapogon) Beiträge zur Entomologie 7 (1/2): 72-73

grisea Petersen, 1973 (Infurcitinea) Beiträge zur Entomologie 23 (1/4): 61

grisea Petersen \& Gaedike, 1982 (Pachyarthra) Fauna of Saudi Arabia 4: 333-334

heydeni Petersen, 1957 (Nemapogon) Beiträge zur Entomologie 7 (1/2): 73; jetzt: Nemapogon inconditella (LuCAS, 1956)

hispanica Petersen \& Gaedike, 1992 (Nemapogon) SHILAP, Revista de Lepidopterologia 20 (80): 333-334

hoenei Petersen \& Gaedike, 1993 (Crypsithyris) Bonner zoologische Beiträge 44 (3/4): 246-247 hoenei Petersen, 1987 (Gerontha) Tinea Supplement 12: 152-153

hoenei Petersen, 1991 (Scalidomia) Deutsche entomologische Zeitschrift N.F. 38 (1/3): 27-31

inornata Petersen, 1966 (Neurothaumasia) Entomologisk Tidskrift 87(1/2): 24; jetzt: Neurothaumasia macedonica Petersen, 1962

intermediella Petersen, 1961 (Catabola) Beiträge zur naturkundlichen Forschung in Südwestdeutschland 20 (1): 64 - 65; jetzt: Perissomastix nigriceps Warren \& Rothschild, 1905

irakella Petersen, 1959 (Paratinea) Bulletin de la Société entomologique d'Égypte 43: 72; jetzt: Ceratobia irakella (Petersen, 1959)

iranensis Petersen, 1964 (Infurcitinea) Beiträge zur naturkundlichen Forschung in Südwestdeutschland 23 (2): 117

iranensis Petersen, 1960 (Morophagoides) Stuttgarter Beiträge zur Naturkunde Nr. 34: 1-2

iranica Petersen \& Gaedike, 1984 (Fermocelina) Acta faunistica entomologica Musei Nationalis Pragae 17 (202): 204; jetzt: Anomalotinea iranica (Petersen \& Gaedike, 1984)

iranica Petersen \& Gaedike, 1984 (Pachyarthra) Acta faunistica entomologica Musei Nationalis Pragae 17 (202): 195-196

islamella Petersen \& Gaedike, 1982 (Neoepiscardia) Fauna of Saudi Arabia 4: 336, 338

japonica Petersen \& Gaedike, 1993 (Crypsithyris) Bonner zoologische Beiträge 44 (3/4): 245-246

kasyi Petersen, 1962 (Infurcitinea) Beiträge zur naturkundlichen Forschung in Südwestdeutschland 21 (2): 216

kasyi Petersen, 1971 (Obesoceras) Beiträge zur Entomologie 21 (3/6): 268; jetzt: Eudarcia kasyi (Petersen, 1971)

kuldjaensis Petersen, 1957 (Ceratophaga) Beiträge zur Entomologie 7 (1/2): 132

lambessella Petersen, 1958 (Infurcitinea) Deutsche Entomologische Zeitschrift N.F. 5 (3/4): 373

lattakianum Petersen, 1968 (Obesoceras) Acta entomologica bohemoslovaca 65 (1): 59-60; jetzt: Eudarcia lattakiana (Petersen, 1968)

leonhardi Petersen, 1957 (Tinea) Beiträge zur Entomologie 7 (1/2): 146; jetzt: Tinea translucens Meyrick, 1917

levantinus Petersen, 1961 (Nemapogon) Časopis Československé Společnosti entomologické 58 (3): 282-283

libanotica Petersen, 1959 (Cephimallota) Deutsche Entomologische Zeitschrift N.F. 6 (1/3): 154-155; jetzt: Cephimallota angusticostella (Zeller, 1839) 
libanoticum Petersen, 1968 (Obesoceras) Acta entomologica bohemoslovaca 65 (1): 60-61; jetzt: Eudarcia holtzi (REBeL, 1902)

litochorella Petersen, 1964 (Infurcitinea) Nachrichtenblatt der Bayerischen Entomologen 13 (3): 22-23

lobata Petersen \& Gaedike, 1979 (Neomeessia) Beiträge zur Entomologie 29 (2): 392; jetzt: Eudarcia lobata (Petersen \& Gaedike, 1979)

luteola Petersen, 1959 (Episcardia) Beiträge zur Entomologie 9 (5/6): 563; jetzt: Edosa exhausta (MEYRICK, 1917)

luteopterella Petersen, 1957 (Reisserita) Beiträge zur Entomologie 7 (1/2): 112-113

macedonica Petersen, 1962 (Neurothaumasia) Beiträge zur naturkundlichen Forschung in Südwestdeutschland 21(2): 206-207

maculata Petersen, 1957 (Lichenotinea) Beiträge zur Entomologie 7 (3/4): 373

maculata Petersen \& Gaedike, 1996 (Neurothaumasia) Entomologische Abhandlungen Staatliches Museum für Tierkunde Dresden 57 (13): 287

maraschensis Petersen, 1968 (Infurcitinea) Acta entomologica bohemoslovaca 65 (1): 62; jetzt: Infurcitinea nigropluviella (WALSINGHAM, 1907)

maroccana Petersen \& Gaedike, 1979 (Fermocelina) Beiträge zur Entomologie 29 (2): 403, jetzt: Anomalotinea. fulvescentella (LuCAs, 1956)

maroccana Petersen \& Gaedike, 1979 (Infurcitinea) Beiträge zur Entomologie 29 (2): 396

marsica Petersen, 1984 (Triaxomera) Reichenbachia, Staatliches Museum für Tierkunde Dresden 22 (18): 141-142

maura Petersen, 1962 (Infurcitinea) The Annals and Magazine of natural History Series 13, 4 (9)(1961): 539

mauretanicus Petersen, 1958 (Rhodobates) Beiträge zur Entomologie 8 (3/4): 403-404; jetzt: Rhodobates algiricellus (REBEL, 1901)

megalopterella Petersen, 1964 (Infurcitinea) Beiträge zur naturkundlichen Forschung in Südwestdeutschland 23 (2): 117

minuscula Petersen \& Gaedike, 1982 (Perissomastix) Fauna of Saudi Arabia 4: 340-341, jetzt: Perissomastix perdita GozManY, 1965

minutella Petersen, 1959 (Haplotinea) Beiträge zur Entomologie 9 (5/6): 567; jetzt: Meneessia minutella (Petersen, 1959)

mongolica Petersen, 1965 (Myrmecozela) Reichenbachia, Staatliches Museum für Tierkunde in Dresden 7 (12): 107-108

montana Petersen, 1957 (Montetinea) Beiträge zur Entomologie 7 (3/4): 344

moreae Petersen \& Gaedike, 1983 (Obesoceras) Annales Musei Goulandris 6: 282-283; jetzt: Eudarcia moreae (Petersen \& Gaedike, 1983)

nana Petersen, 1973 (Paratinea) Beiträge zur Entomologie 23 (1/4): 68; jetzt: Metatinea nana (Petersen, 1973)

nepalensis Petersen, 1982 (Episcardia) Reichenbachia, Staatliches Museum für Tierkunde in Dresden 20 (8): 73; jetzt: Eudarcia opsigona (MEYRICK, 1911)

nigrocapitella Petersen, 1959 (Morophaga) Beiträge zur Entomologie 9 (5/6): 571-572; jetzt: Morophaga cremnarcha (MEYRICK, 1932) 
nigrocephala Petersen, 1982 (Perissomastix (Lazocatena)) Reichenbachia, Staatliches Museum für

Tierkunde in Dresden 20 (8): 73-74; jetzt: Lozocatena nigrocephala (Petersen, 1982)

nuristanica Petersen, 1963 (Infurcitinea) Beiträge zur Entomologie 13 (1/2): 185-186

obscura Petersen, 1959 (Catabola) Beiträge zur Entomologie 9 (5/6): 560; jetzt: Lozocatena obscura (Petersen, 1959)

obscura Petersen, 1973 (Infurcitinea) Beiträge zur Entomologie 23 (1/4): 61

obscuroides Petersen \& Gaedike, 1984 (Infurcitinea) Acta faunistica entomologica Musei Nationalis Pragae 17 (202): 192; jetzt: Infurcitinea obscuroides GAEDIKe, 1983

ochridella Petersen, 1962 (Infurcitinea) Beiträge zur naturkundlichen Forschung in Südwestdeutschland 21 (2): 215

olympica Petersen, 1958 (Infurcitinea) Deutsche Entomologische Zeitschrift N.F. 5 (3/4): 372

oranella Petersen, 1957 (Fermocelina) Beiträge zur Entomologie 7 (1/2): 120-121; jetzt: Reisserita oranella (Petersen, 1957)

orientale, ssp. von petrinellum PetERSEN, 1973 (Ateliotum) Beiträge zur Entomologie 23 (1/4): 65

orientalis Petersen, 1961 (Nemapogon) Časopis Československé Společnosti entomologické 58 (3): 281-282

orientalis Petersen, 1959 (Paratinea) Beiträge zur Entomologie 9 (5/6): 569-570; jetzt: Proterospastis orientalis (Petersen, 1959)

paghmanella Petersen, 1973 (Episcardia) Beiträge zur Entomologie 23 (1/4): 64; jetzt: Edosa paghmanella (Petersen, 1973)

parentii Petersen, 1964 (Infurcitinea) Reichenbachia, Staatliches Museum für Tierkunde in Dresden 2 (58): 233

parva Petersen \& Gaedike, 1979 (Reisserita) Beiträge zur Entomologie 29 (2): 402

parvum Petersen, 1988 (Ateliotum) Beiträge zur Entomologie 38 (2): 314

pinkeri Petersen, 1987 (Rhodobates) Entomologische Abhandlungen Staatliches Museum für Tierkunde Dresden 50 (9): 173-174

pseudoranella Petersen \& Gaedike, 1979 (Reisserita) Beiträge zur Entomologie 29 (2): 401-402 punctata Petersen, 1961 (Hapsifera) Beiträge zur naturkundlichen Forschung in Südwestdeutschland 20 (1): 63-64

pygmaeana Petersen, 1959 (Episcardia) Beiträge zur Entomologie 9 (5/6): 564; jetzt: Edosa pygmaeana Petersen, 1959

quettaella Petersen, 1971 (Infurcitinea) Beiträge zur Entomologie 21 (3/6): 268-270

raddei Petersen, 1958 (Infurcitinea) Deutsche Entomologische Zeitschrift N.F. 5 (3/4):370-371

reisseri Petersen, 1968 (Infurcitinea) Acta entomologica bohemoslovaca 65 (1): 63

reisseri Petersen \& Gaedike, 1983 (Nemapogon) Annales Musei Goulandris 6: 276-277

rhenania Petersen, 1962 (Lichenovora) The Annals and Magazine of natural History Series 13, 4

(9)(1961): 533-534; jetzt: Tenaga rhenania (Petersen, 1962)

romanum Petersen, 1958 (Obesoceras) Deutsche Entomologische Zeitschrift N.F. 5 (3/4): 368-369; jetzt: Eudarcia romana (Petersen, 1958)

rostrata Petersen, 1966 (Tinea) Entomologisk Tidskrift 87 (1/2): 247

safedella Petersen, 1973 (Infurcitinea) Beiträge zur Entomologie 23 (1/4): 59-60 
sarobiella Petersen, 1959 (Catabola) Beiträge zur Entomologie 9 (5/6): 561; jetzt: Perissomastix paramima (MeYrick, 1917)

sarobiella Petersen, 1959 (Paratinea) Beiträge zur Entomologie 9 (5/6): 570; jetzt: Crypsithyris sarobiella (PETERSEN, 1959)

semifulvelloides Petersen, 1973 (Tinea) Beiträge zur Entomologie 23 (1/4): 65-66

siciliana Petersen, 1964 (Infurcitinea) Nachrichtenblatt der Bayerischen Entomologen 13 (3): $21-22$

signatellus Petersen, 1957 (Nemapogon) Beiträge zur Entomologie 7 (1/2): 80-81

sinensis Petersen \& Gaedike, 1993 (Niditinea) Bonner zoologische Beiträge 44 (3/4): 244-245

sinensis Petersen, 1987 (Rhodobates) Entomologische Abhandlungen Staatliches Museum für Tierkunde Dresden 50 (9): 174

splendens Petersen, 1973 (Episcardia) Beiträge zur Entomologie 23 (1/4): 63 - 64; jetzt: Edosa nestoria (MEYRICK, 1910)

steueri Petersen, 1966 (Tinea) Entomologische Nachrichten 1966 (2): 35

striaticostella Petersen, 1959 (Paratinea) Bulletin de la Société entomologique d'Égypte 43: 71-72; jetzt: Wyoma striaticostella (Petersen, 1959)

subtile Petersen, 1973 (Obesoceras) Beiträge zur Entomologie 23 (1/4): 58; jetzt: Eudarcia subtilis (Petersen, 1973)

tauridella Petersen, 1968 (Infurcitinea) Acta entomologica bohemoslovaca 65 (1): 62-63

teheranensis Petersen, 1971 (Infurcitinea) Beiträge zur Entomologie 21 (3/6): 268

tihamaella Petersen \& Gaedike, 1982 (Perissomastix) Fauna of Saudi Arabia 4: 341

trapezoides Petersen \& Gaedike, 1993 (Monopis) Bonner zoologische Beiträge 44 (3/4): 247-248

trimaculata Petersen, 1973 (Paratinea) Beiträge zur Entomologie 23 (1/4): 67-68; jetzt: Crypsithyris trimaculata (Petersen, 1973)

turcica Petersen, 1968 (Infurcitinea) Acta entomologica bohemoslovaca 65 (1): 63-64

vartianae Petersen, 1962 (Infurcitinea) Beiträge zur naturkundlichen Forschung in Südwestdeutschland 21 (2): 219

walsinghami Petersen, 1962 (Infurcitinea) The Annals and Magazine of natural History Series 13, 4 (9)(1961): 537

wiltshirella Petersen, 1964 (Catabola) Beiträge zur naturkundlichen Forschung in Südwestdeutschland 23 (2): 115; jetzt: Perissomastix wiltshirella (Petersen, 1964)

xerxes Petersen \& Gaedike, 1984 (Episcardia) Acta faunistica entomologica Musei Nationalis Pragae 17(202): 197 - 198; jetzt: Edosa xerxes (Petersen \& Gaedike, 1984)

zernyi Petersen, 1957 (Catabola) Beiträge zur Entomologie 7 (5/6): 568; jetzt: Crassicornella zernyi (Petersen, 1957)

zernyi Petersen, 1957 (Reisserita) Beiträge zur Entomologie 7 (1/2): 110

R. GAEDIKE 